\title{
Aplicação da Prospecção Tecnológica no Estudo de Caso da Palmilha Sensorizada para Pés Diabéticos
}

\section{Application of Technological Prospection in the Case Study of the Insole With Diabetic Feet Sensors}

\author{
Luciana Maria de Oliveira Cortinhas ${ }^{1}$ \\ Leonara Gonçalves e Silva Pires ${ }^{1}$ \\ Anna Patrícia Teixeira Barbosa ${ }^{1}$ \\ Jeane Souza Chaves Sidou ${ }^{1}$ \\ Camila Alves Areda ${ }^{1}$ \\ Paulo Gustavo Barboni Dantas Nascimento ${ }^{1}$ \\ Rafael Leite Pinto de Andrade ${ }^{1}$ \\ ${ }^{1}$ Universidade de Brasília, Brasília, DF, Brasil
}

\begin{abstract}
Resumo
O presente artigo realiza uma prospecção tecnológica do pedido de patente intitulado "palmilha sensorizada para pés diabéticos" de titularidade da Fundação Universidade de Brasília, com o intuito de analisar a viabilidade de sua manutenção em razão do cenário atual de crise orçamentária. Foram mapeadas tecnologias similares, no Brasil e no Exterior, que utilizam sinais bioelétricos, no corpo ou em suas partes, para monitoramento. A busca por patentes se deu na base Orbit. Também foram analisadas publicações sobre sistemas eletrônicos de monitoramento, nas bases de dados das plataformas Scopus e Web of Science. Identificou que a área apresentou crescimento nos últimos anos, porém que os estudos ainda são incipientes. O trabalho visou também a avaliação da maturidade tecnológica do invento, pelo que concluiu que o grau de prontidão tecnológica da palmilha estudada é TRL 5.
\end{abstract}

Palavras-chave: Sinais bioelétricos. Corpo. Palmilha.

\begin{abstract}
The present article carries out a technological prospection of the technology titled "sensorized footbed for diabetic feet", owned by the University of Brasilia Foundation, in order to analyze the viability of maintaining its patent application due to the current scenario of budget crisis. Similar technologies have been mapped, in Brazil and abroad, that use bioelectrical signals, in the body or in its parts, for monitoring. The search for patents was based on Orbit. We also analyzed publications on electronic monitoring systems in the databases of the Scopus and Web of Science platforms. It was identified that the area presented growth in the last years, but that the studies are still incipient. The study also aimed at evaluating the technological maturity of the invention, so it was concluded that the degree of technological readiness of the insole studied is TRL 5.
\end{abstract}

Keywords: Bioelectrical signals. Body. Insole.

Áreas tecnológicas: Prospecção tecnológica. Transferência de tecnologia. 


\section{Introdução}

Dados da pesquisa de Vigilância de Fatores de Risco e Proteção para Doenças Crônicas por Inquérito Telefônico (Vigitel) do Ministério da Saúde indicam que de 2006 a 2016, o número de brasileiros com diabetes aumentou $61,8 \%$. Isso significa que a doença passou de $5,5 \%$ da população para 8,9\% (BRASIL, 2017).

Entretanto, poucos produtos nacionais são desenvolvidos com o intuito de melhorar a qualidade de vida do paciente diabético. Segundo Andreassi e Sbragia (2002), no Brasil, diferente do que ocorre em outros países, são os resultados de vendas que condicionam os investimentos futuros em Pesquisa e Desenvolvimento (P\&D). Era esperado que os investimentos em $\mathrm{P} \& \mathrm{D}$ fossem futuramente compensados com a participação dos novos produtos na receita total das empresas.

Nesse contexto, destacam-se trabalhos realizados em universidades brasileiras, como a "palmilha sensorizada para pés diabéticos", de titularidade da Fundação Universidade de Brasília (FUB). O pedido de patente depositado no Instituto Nacional da Propriedade Industrial (INPI), sob o registro PI 1103692-5, em 18 de julho de 2011, encontra-se em análise já há sete anos. A invenção tem como princípio detectar e monitorar os principais pontos de pressão do pé, de modo a realizar um estudo mais detalhado sobre as áreas suscetíveis ao desenvolvimento de feridas em pés de pessoas com diabetes. A tecnologia visa principalmente ao estágio de prevenção, mas sem deixar de atender aos pacientes acometidos por feridas. A palmilha segue a anatomia e as características dos pés do usuário e é confeccionada, de forma individualizada e personalizada, em látex hipoalergênico, proporcionando maior conforto e bem-estar.

Entretanto, mesmo sendo um dos grandes polos de pesquisa no Brasil, as universidades têm dificuldade em manter os pedidos de patentes, uma vez que há custos envolvidos e, com a nova realidade imposta pela Emenda Constitucional n. 95/2016 (BRASIL, 2016), limitando os gastos públicos, uma nova era de eficiência e planejamento de gastos deve ser estabelecida.

Nesse contexto, o presente estudo visa a identificar o mercado mundial em que a tecnologia "palmilha sensorizada para pés diabéticos" encontra-se inserida. Pretende-se embasar uma futura discussão sobre a manutenção ou não do pedido de proteção da patente e suporte dos gastos diretos do Centro de Apoio do Desenvolvimento Tecnológico (CDT/UnB), efetuados ao INPI a título de taxas.

No presente trabalho, é apresentada uma prospecção, de abrangência nacional e internacional, da tecnologia de titularidade da FUB, detectando inventos similares, publicações sobre o assunto, viabilidade comercial bem como grau de prontidão tecnológica.

Portanto, a intenção principal é, considerando o histórico do pedido de patente e o tempo decorrido para análise, verificar qual é o atual cenário no qual se localiza a tecnologia prospectada. E, por fim, realizar uma qualificação tecnológica do pedido de responsabilidade do $\mathrm{CDT} / \mathrm{UnB}$ frente a um cenário mundial e regional, fornecendo subsídios a uma proposta de gerenciamento de ativos de Propriedade Industrial pela UnB. 


\section{Metodologia}

A metodologia de pesquisa adotada, no presente estudo, permitiu a avaliação do cenário mundial e nacional de tecnologias de sistemas de monitoramento eletrônicos, a partir de informações obtidas em bases de patentes e de artigos, nacionais e internacionais. Para tanto, foram escolhidas as plataformas Orbit, Scopus e Web of Science.

Foi realizada a análise bibliométrica quantitativa acerca do tema que envolve a tecnologia analisada. Essa é uma técnica para o mapeamento dos principais autores, periódicos e palavras-chave sobre determinado tema (PRITCHARD, 1969). Ela examina o material bibliográfico de uma perspectiva objetiva, quantitativa, que é útil para organizar a informação em um campo temático específico (MERIGÓ; GIL-LAFUENTE; YQGER, 2015). É também uma forma de análise de publicações científicas que avalia a evolução do conhecimento de um assunto específico, a qualidade científica e a influência de obras e fontes (BOUYSSOU; MARCHANT, 2011; DAIM et al., 2006).

A análise bibliométrica seguiu seis etapas: i) definição do campo de estudo; ii) escolha do banco de dados; iii) ajuste dos critérios de busca; iv) compilação das categorias de informações bibliográficas; v) codificação do material recuperado e, finalmente; e vi) análise da informação.

Foram utilizadas diferentes combinações de palavras-chave, nos campos "título" e "resumo", a fim de melhor representar o objeto de pesquisa. As palavras-chave utilizadas foram as seguintes: "bioelec", "signal" e "body". Para melhor descrever a tecnologia, foram usadas técnicas clássicas de pesquisa, como operadores booleanos ("and" e "or"), de truncamento (*) e sinônimos para as palavras-chave ("bio-signal").

Os resultados obtidos foram analisados a fim de identificar os principais países de prioridade das tecnologias, as empresas mais atuantes nessa área, a evolução do número de proteções ao longo dos últimos anos, a distribuição dos depósitos em universidades e a situação atual das proteções. Com relação às publicações científicas, foram identificados os principais autores, universidades, revistas, países, áreas temáticas e períodos de ascendência do tema.

Posteriormente, o estudo foi direcionado para a obtenção do grau de maturidade da tecnologia "palmilha sensorizada para pés diabéticos". O nível de prontidão tecnológica foi obtido considerando a escala Technology Readiness Level (TRL).

\section{Resultados e Discussão}

\subsection{Mapeamento de Inventos na Base de Patentes da Plataforma Orbit}

A prospecção tecnológica é uma ferramenta capaz de mapear sistematicamente os desenvolvimentos científico e tecnológico, os quais, por sua vez, são capazes de influenciar de forma significativa os investimentos em tecnologia, que impactam diretamente na indústria, na economia e nas relações de consumo presentes na sociedade como um todo (SERAFINI et al., 2012).

No dia 14/06/2018, foi realizada uma pesquisa de depósitos de patentes na base Orbit, com filtro para intervalo de 20 anos (1997-2017). As buscas foram iniciadas, no título e resumo, com as palavras-chave "bioelec*" e "bio-signal" com o operador booleano "or" e o truncamento 
(*), tendo sido obtidos 3.351 resultados. Para o aprimoramento, foi inserida a palavra-chave "signal" com o operador booleano "and", resultando 1.678 registros. Por fim, foi adicionada a palavra-chave "body", também com o operador booleano "and", obtendo o resultado de 616 registros.

Optou-se pela não utilização da palavra-chave "insole" (palmilha) tendo em vista que restringia muito a busca e inviabilizava a análise de mercado da tecnologia objeto deste estudo. Atentou-se também ao fato de que o pedido de patente reivindicado não é somente para uma palmilha, mas também para o sistema de monitoramento de pressão plantar. Os dados estão demonstrados na Tabela 1.

Tabela 1 - Resultados da busca na base de patentes da plataforma Orbit para as palavras-chave "bioelec*" or "bio-signal" and "signal" and "body", 2018

\begin{tabular}{ccc} 
Busca avançada & Palavras-chave & Resultados \\
$1^{\mathrm{a}}$ & "bioelec*" or "bio-signal" & 3.351 \\
$2^{\mathrm{a}}$ & ("bioelec" or "bio-signal") and "signal" & 1.678 \\
$3^{\circ}$ & ("bioelec*" or "bio-signal") and "signal" and "body" & 616 \\
\hline
\end{tabular}

Fonte: Elaborada pelos autores deste artigo (2018)

Os 616 resultados encontrados foram selecionados para confecção de gráficos, que possibilitaram as análises que serão a seguir expostas.

Figura 1 - Patentes disponíveis na base de patentes da plataforma Orbit para as palavras-chave "bioelec" or "bio-signal" and "signal" and "body", por ano de publicação

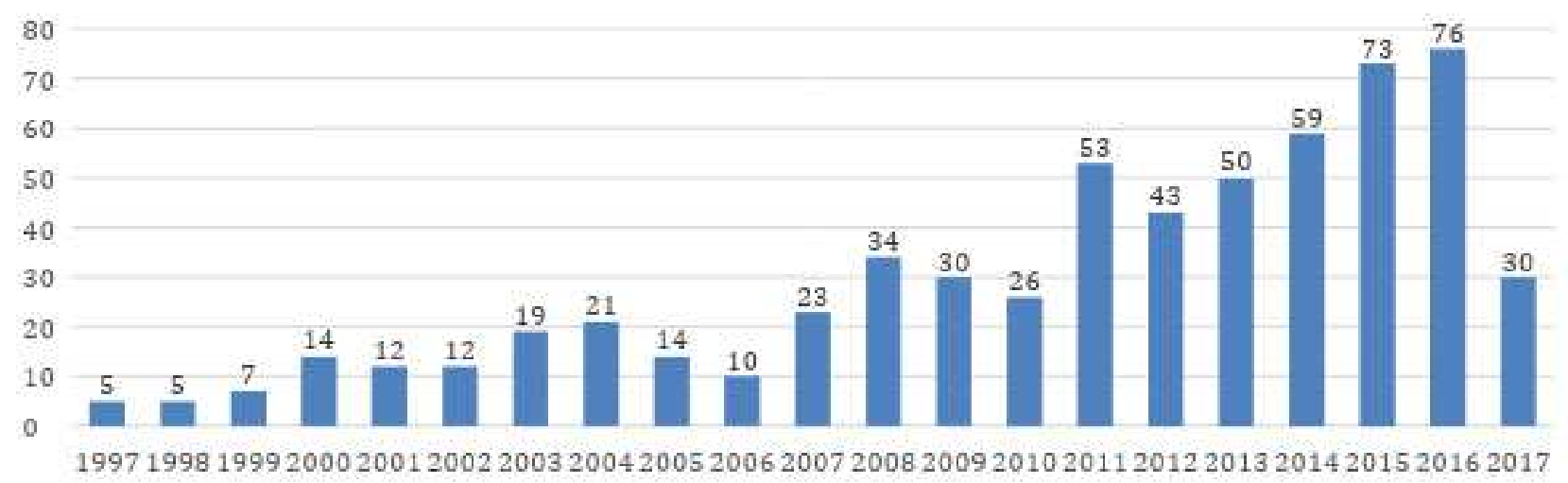

Fonte: Elaborada pelos autores deste artigo (2018)

Nota-se que o número de patentes teve uma evolução expressiva nos últimos dez anos, em especial nos anos de 2015 e 2016. Há uma redução no ano de 2017, provavelmente por causa do período de sigilo exigido pela lei brasileira que é de 18 meses, portanto, as patentes ainda não estão disponíveis para consulta.

Salienta-se que os resultados selecionados representam tecnologias que detectam, medem ou registram sinais bioelétricos do corpo ou de suas partes, conforme a classificação do pedido de patente analisado (IPC A61B5/04). 
Foi possível observar, que o país com maior número de depósitos de patentes com a tecnologia de sensores para detectar e monitorar pontos de pressão no corpo humano é a República da Coréia, que totaliza 373 registros, quantidade superior à soma dos registros de todos os outros 15 países subsequentes do ranking de depositantes para essa família tecnológica (China, Japão, Estados Unidos, entre outros).

Pela Figura 2, pode-se verificar que a tecnologia aplicada no pedido de patente objeto deste estudo é encontrada em diversos segmentos, porém os mais relevantes são ligados aos temas médicos-biológicos, com o objetivo do monitoramento dos sinais do corpo e níveis de atividade e suas intensidades.

Figura 2 - Principais nichos tecnológicos para tecnologias de sensores para detectar e monitorar pontos de pressão no corpo humano segundo patentes disponíveis na base de patentes da plataforma Orbit

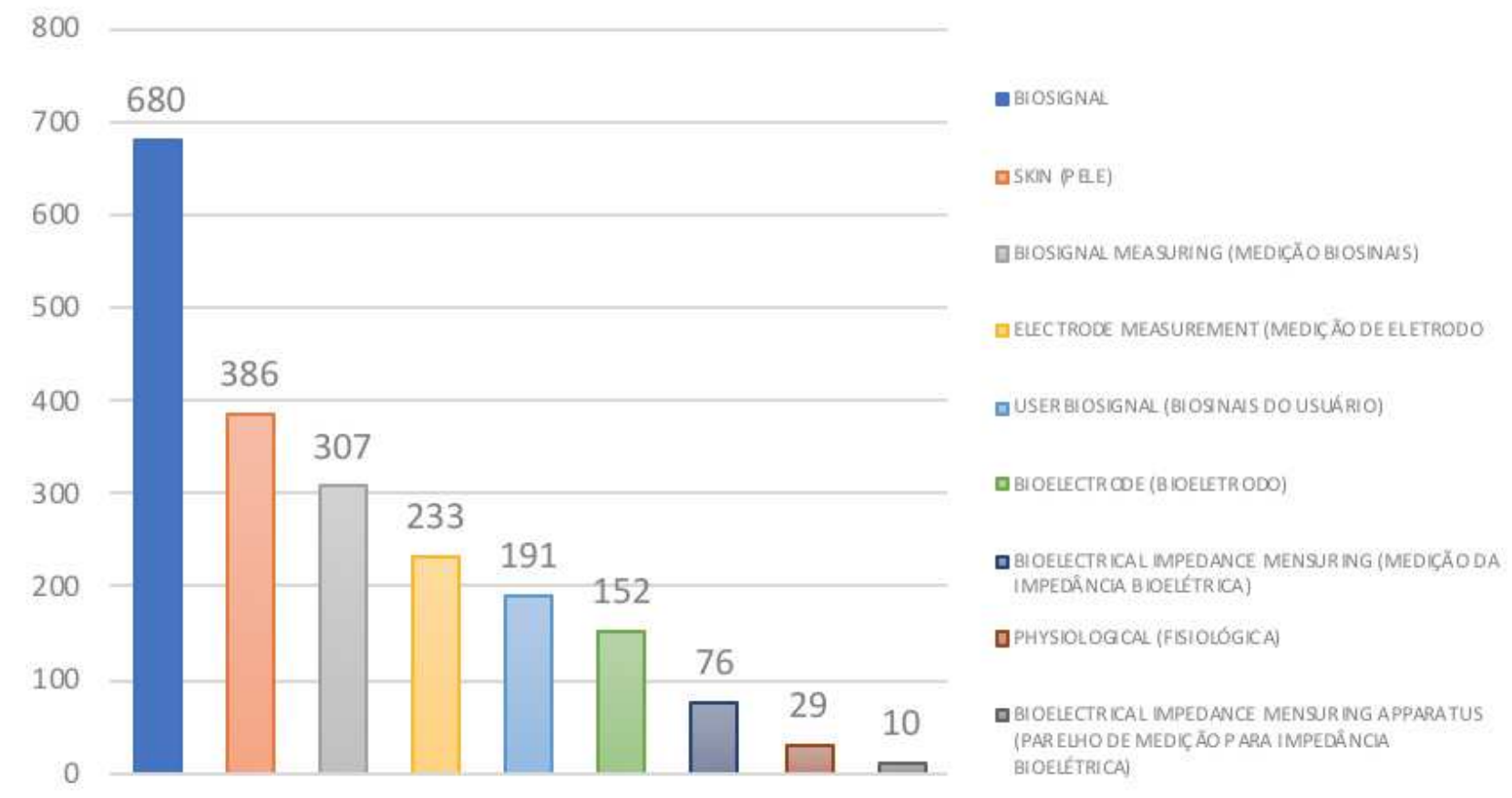

Fonte: Elaborada pelos autores deste artigo (2018)

Cabe esclarecer que a mesma patente pode se enquadrar em mais de um nicho tecnológico, motivo pelo qual pode ser observada a quantidade apresentada na Figura 2 em cada segmento.

Na Figura 3, é confirmada a liderança da República da Coréia como território propício para o investimento em tecnologia e inovação, pois, das 16 maiores instituições depositantes de patentes apenas cinco não se encontram no território sul-coreano.

A empresa Samsung destaca-se como maior depositante no período de 20 anos pesquisado, que é de 1997 a 2017. Somando-se o percentual de depósitos das principais empresas sul-coreanas obtém-se um percentual superior a $60 \%$ do total de registros ligados à tecnologia.

Pode ser observado que a Samsung detém mais de $25 \%$ de todos os registros de pedidos e concessões de patentes ligadas à tecnologia de sensores e monitoramento. 
Figura 3 - Principais depositantes da tecnologia de sensores para detectar e monitorar pontos de pressão no corpo humano segundo patentes disponíveis na base de patentes da plataforma Orbit

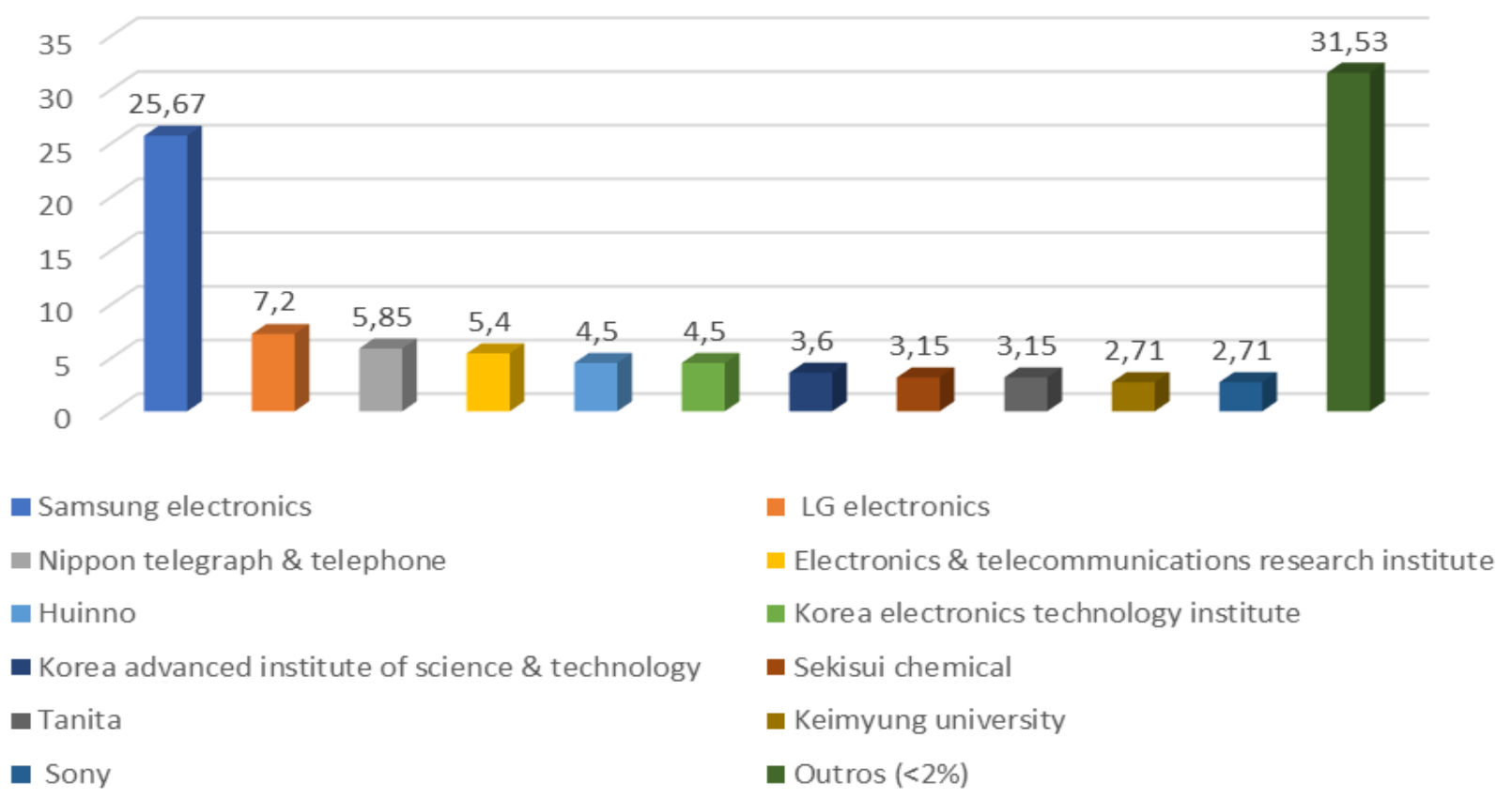

Fonte: Elaborada pelos autores deste artigo (2018)

\subsection{Mapeamento de Publicações nas Bases de Dados das Plataformas Scopus e Web of Science}

No dia 13/06/2018, na base Scopus foi realizada uma busca no título, resumo e palavras-chave de documentos que contivessem (("biolec*" or "bio-signal") and "signal" and "body"), com filtro para publicações de 1997 a 2017. A utilização das mesmas palavras-chave e período utilizado para a busca de patentes foi proposital. A busca refinada resultou em 228 documentos, os quais serviram para a análise.

A pesquisa realizada na plataforma Web of Science, em 12/06/2018, que utilizou a mesma combinação das palavras-chave, os mesmos ordenadores booleanos e delimitação de período, possibilitando inicialmente a localização de resultados bem abrangentes, porém, com o refinamento da busca foi possível chegar a um número de publicações aproximado ao da outra base, conforme descrito na Tabela 2.

Tabela 2 - Busca por palavras-chave e demais combinações e os respectivos resultados nas bases de dados das plataformas Scopus e Web of Science para as palavras-chave "bioelec*" or "bio-signal" and "signal" and "body", 2018

$\begin{array}{cccc} & \text { Palavras-chave } & \text { Resultados Scopus } & \text { Resultados Web of Science } \\ 1^{\text {a }} & \text { ("biolec*" or "bio-signal") } & 1.381 & 6.606 \\ 2^{\mathrm{a}} & \text { (("biolec*" or "bio-signal") and "signal" } & 1.286 & 1605 \\ 3^{\mathrm{a}} & (\text { ("biolec*" or "bio-signal") and } & 228 & 194\end{array}$

Fonte: Elaborada pelos autores deste artigo (2018) 
A distribuição das patentes por ano de publicação permite identificar uma evolução o número de depósitos nos últimos anos, conforme a Figura 4.

Figura 4 - Documentos disponíveis na base da plataforma Web of Science para as palavras-chave "bioelec" or "bio-signal" and "signal" and "body", por ano de publicação

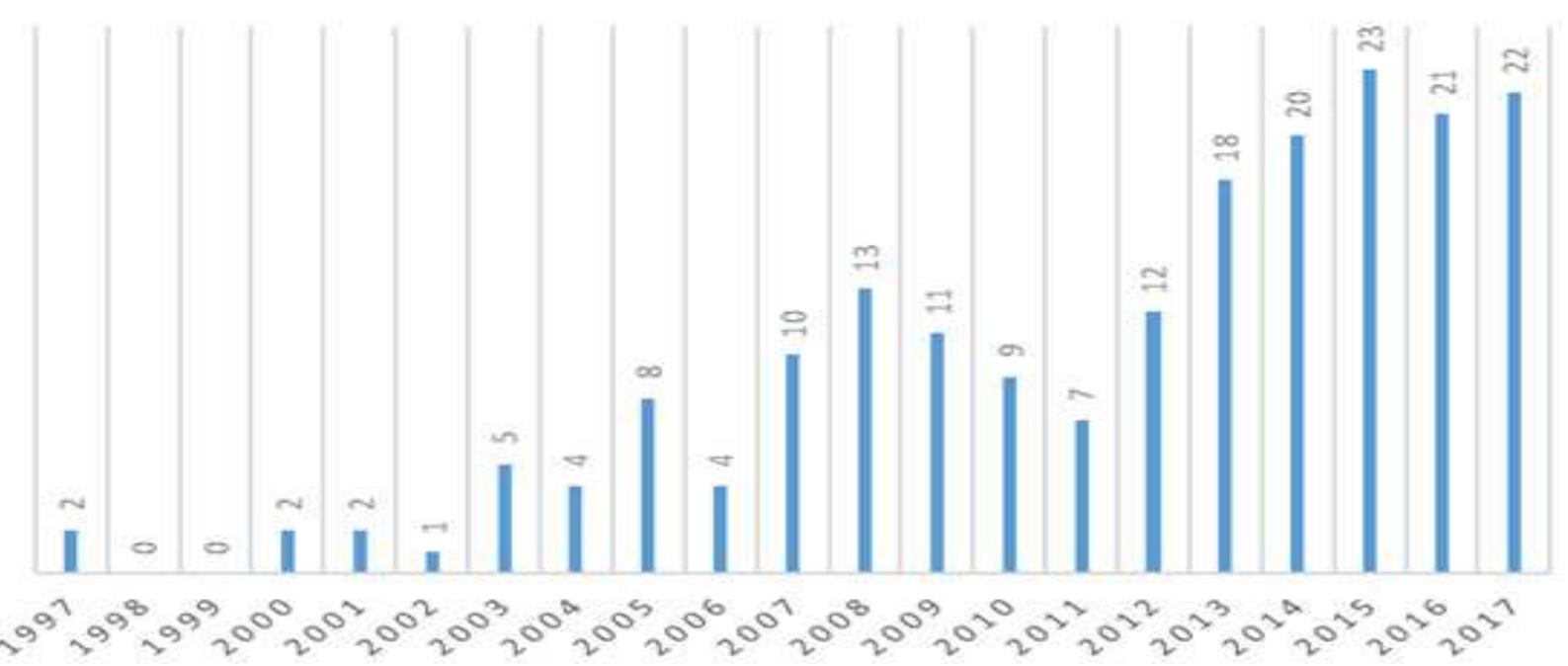

Fonte: Elaborada pelos autores deste artigo (2018)

É possível notar que, nos últimos 20 anos, houve um aumento importante de publicações sobre o assunto, em especial de 2013 a 2017, com leve decréscimo em 2016. A base de dados Scopus indicou o ápice de publicações sobre o tema no ano de 2014 e a base Web of Science no ano de 2015.

Entre as cinco fontes com mais publicações sobre o tema, duas são do Instituto de Engenheiros Eletricistas e Eletrônicos (IEEE). Essa organização foi responsável por $14 \%$ das publicações sobre o tema analisado. O IEEE se considera a maior organização profissional técnica do mundo dedicada ao avanço da tecnologia em benefício da humanidade. Declaram que seu objetivo principal é promover a inovação e a excelência tecnológica para o benefício da humanidade.

O IEEE é liderado por um grupo diversificado de membros voluntários eleitos e nomeados. A estrutura de governança inclui conselhos para áreas operacionais bem como órgãos que representam membros das 46 sociedades e conselhos técnicos e dez regiões geográficas mundiais.

$\mathrm{Na}$ base Web of Science a apresentação do demonstrativo das publicações em periódicos científicos, ofereceu destaque para a revista americana Bioelectromagnetics (BEM), que publicou $15 \%$ de artigos sobre o tema. A BEM serve como um ponto focal para cientistas, profissionais, acadêmicos, estudantes de pós-graduação, bolsistas e associados que buscam pesquisas em todo o mundo. A revista publica artigos e pesquisa de alta qualidade, com foco em aspectos experimentais, teóricos e clínicos da interação e aplicação de campos eletromagnéticos e ondas em sistemas biológicos.

Os líderes em publicações, nas duas bases pesquisadas, são os autores Giovanni Ansaloni e David Atienza Alonso, porém, aparecem em posições trocadas. David em primeiro lugar na base Scopus e Giovanni na base Web of Science.

Giovanni Ansaloni é pesquisador de pós-doutorado na Faculdade de Informática da Universitá della Svizzera Italiana (USI-Lugano). Sua pesquisa atual é centrada na customização arquitetural de domínio específico, estudando novas estratégias para a automação de identifica- 
ção e síntese de extensão de conjunto de instruções e a exploração de matrizes reconfiguráveis de granulação grossa específicas de domínio. Essas duas linhas de pesquisa são fundadas por dois subsídios separados do SNF. De 2011 a 2015, trabalhou como pós-doc no Laboratório de Sistemas Embarcados da École Polytechnique Fédérale de Lausanne (ESL-EPFL). Coordenou os esforços de pesquisa com foco nos sensores sem fio inteligentes, incluindo otimizações de software de algoritmos de processamento para análise de sinal biológico e explorações arquitetônicas de plataformas WBSN de baixíssimo consumo de energia.

David Atienza Alonso professor associado de engenharia elétrica e de computação e diretor do Laboratório de Sistemas Embarcados (ESL) no Instituto de Engenharia Elétrica da Escola de Engenharia (STI) da Escola Federal Politécnica de Lausanne (EPFL), Suíça. Anteriormente, foi professor associado do Departamento de Arquitetura e Automação de Computadores da Universidade Complutense de Madri (UCM), Espanha. Ele recebeu seu M.Sc. e doutorado em Ciência da Computação pela Universidade Complutense de Madrid, Espanha, e pelo Centro Interuniversitário de Microeletrônica (IMEC), Leuven, Bélgica, em 2001 e 2005, respectivamente.

Dos 228 documentos pesquisados na plataforma Scopus, pode-se observar que foram originados na maioria em instituições de ensino da Suíça, Coréia do Sul, Itália e Portugal. A Escola Federal Politécnica de Lausanne (EPFL) apareceu em primeiro lugar com grande vantagem no número de documentos produzidos sobre as demais.

A análise por países, na base Scopus, confirma a predominância da Coréia do Sul (37 documentos), da mesma forma como ocorre nas patentes, seguida logo depois da Índia (33), Estados Unidos (27), Japão (19), Suíça (16), China (12), Itália (12) Taiwan (11) e Canadá (9). O Brasil aparece com apenas um documento.

Já na base Web of Science, verificou-se a predominância dos Estados Unidos (38) seguido de Índia (21), Coréia do Sul (20), China (16), Itália (12), Taiwan (11), Japão (10) e Suíça (9).

Figura 5 - Documentos disponíveis na base da plataforma Web of Science para as palavras-chave "bioelec" or "bio-signal" and "signal" and "body", por países base

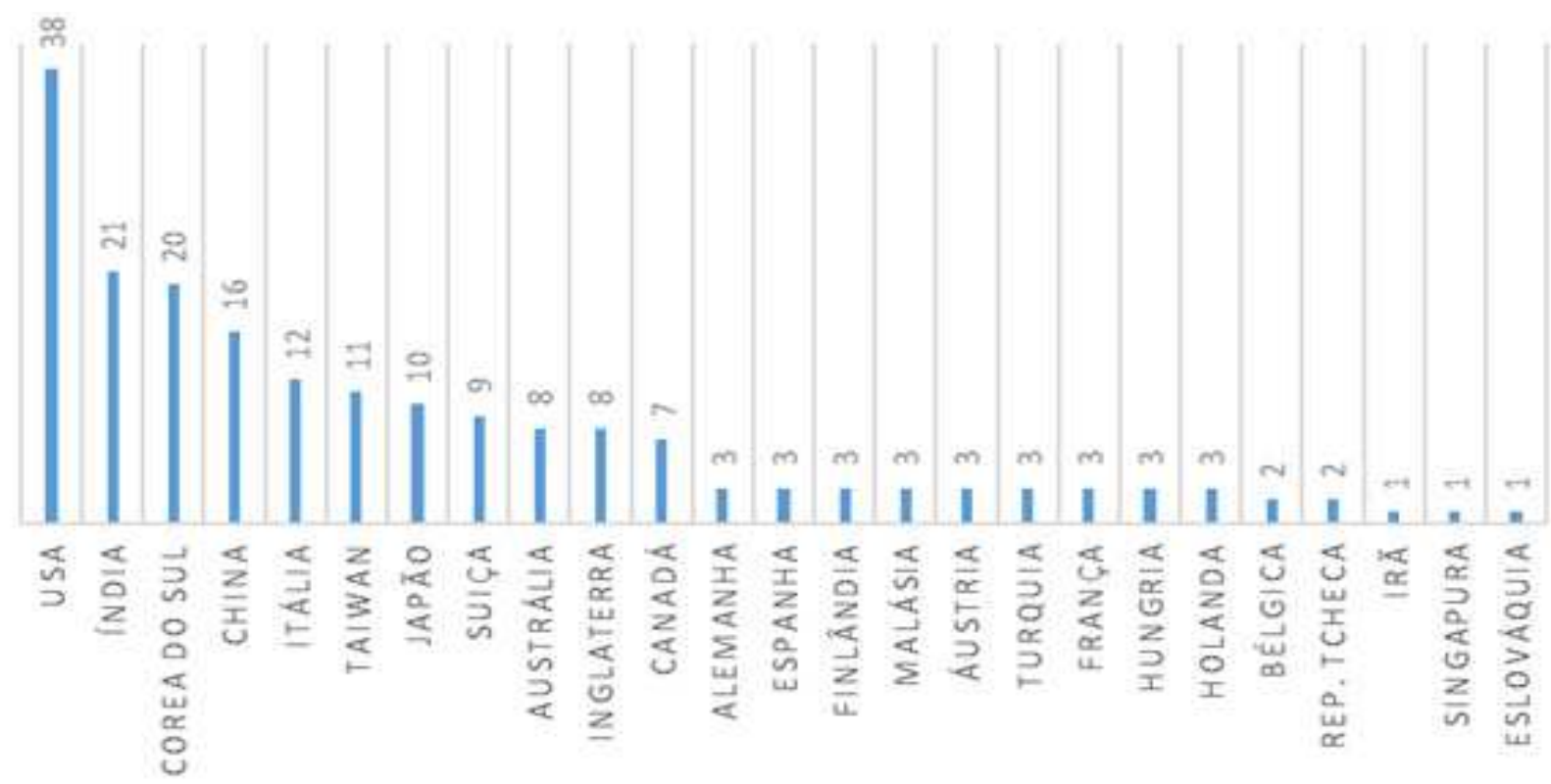

Fonte: Elaborada pelos autores deste artigo (2018) 
A Figura 5 representa o volume de publicações do tema pesquisado com destaque para os Estados Unidos e a Índia que juntos somam $30 \%$ do material produzido ao longo do período avaliado.

Não foi identificado nenhum autor brasileiro, mas o cenário no Brasil está bastante favorável. No relatório produzido pela Clarivate Analytics para a Capes, publicado em janeiro de 2018, foi desenvolvida uma análise do panorama da produção científica do Brasil. A Clarivate fez uso da bibliometria para analisar documentos de pesquisas brasileiros publicados de 2011 a 2016, tendo sido identificados pontos fortes e oportunidades para a política de pesquisa $e$ ciência brasileira, concluindo que "O Brasil é o $13^{\circ}$ maior produtor de publicações de artigos em nível mundial e seus resultados de pesquisa crescem anualmente." (CROSS; THOMSON; SIBCLAIR, 2018).

A Revista Nature, publicação de fevereiro de 2018, apontou que existe uma tendência entre os países em desenvolvimento em um maior investimento em ciência e tecnologia e consequentemente um aumento da produção científica, mas ressaltam que "Os Estados Unidos continuam sendo uma potência científica mundial, com pesquisas de alto nível de qualidade e impacto, formando o maior de número de doutores em ciência e tecnologia, além de ser, ainda, o principal destino de pesquisadores internacionais." (NATURE, 2018).

Dos 228 documentos analisados na base Scopus, verificou-se que 143 são papéis de conferência $(62,7 \%)$ e 77 são artigos (33,8\%). Assim, vê-se que embora o tema venha sendo bastante pesquisado, divulgado em conferências e compartilhado com os pares, o fato de constar mais em papéis de conferência do que em artigo indica que é uma área que ainda precisa ser consolidada cientificamente.

Com relação à área de estudo, a pesquisa demonstrou ser marcante a predominância do assunto em Engenharia (143 documentos - 62,7\%), seguida de Ciência da computação (129 $-56,6 \%)$ e Medicina (52 - 22,8\%).

Figura 6 - Documentos disponíveis na base da plataforma Web of Science para as palavras-chave "bioelec*" or "bio-signal" and "signal" and "body", por número de citações base Web of Science

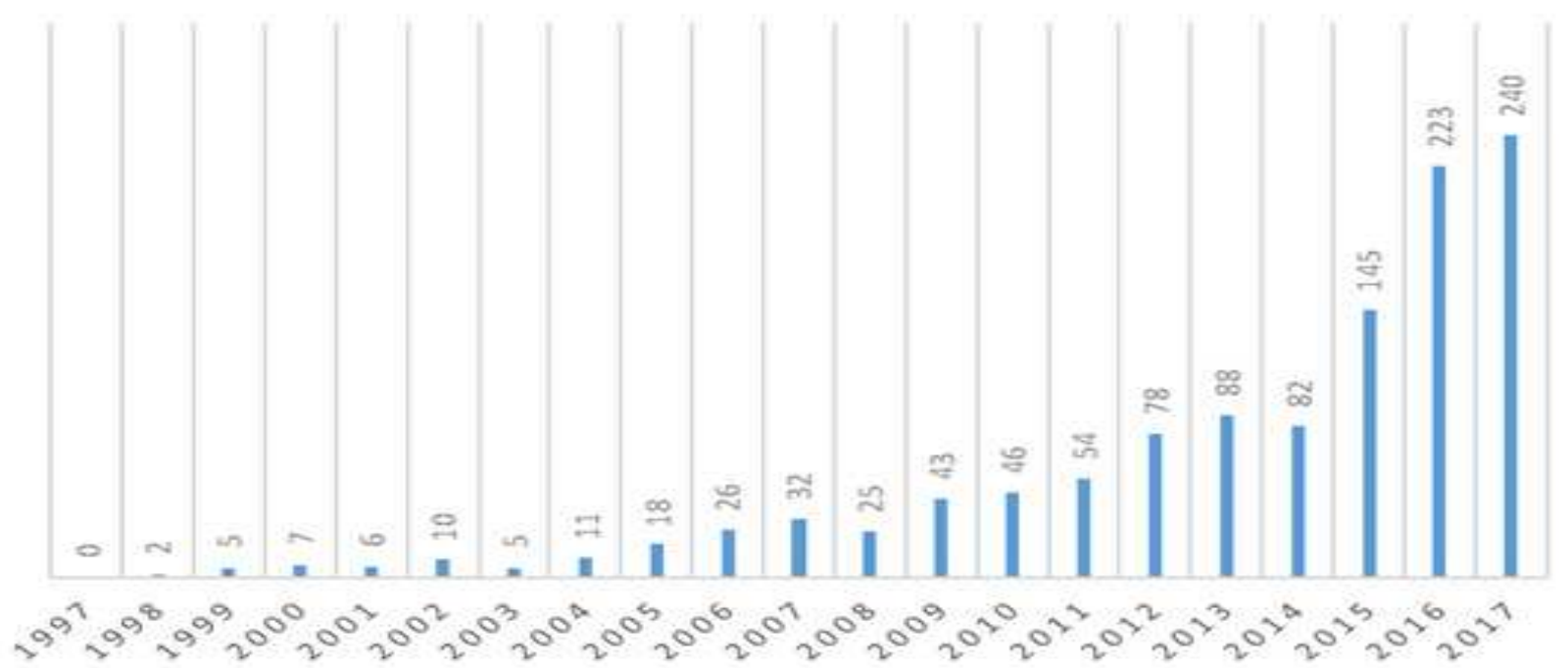

Fonte: Elaborada pelos autores deste artigo (2018) 
A Figura 6 demonstra o número de citações sobre o tema. Foram 1.242 com uma média de 59,14 por ano, somente no ano de 2017 foram registradas 250 citações.

Segundo Di Cross, Simon Thomson e Alexandra Sinclair, "O número de citações que uma publicação de pesquisa (paper) recebe reflete o impacto que teve em pesquisas posteriores. As publicações científicas citam documentos anteriores para validar uma contribuição intelectual". Assim, torna-se possível dizer que uma publicação (ou uma coleção de publicações) que tenha uma contagem de citações mais elevada teve também um impacto maior no campo de conhecimento ao qual se relacionou (CROSS; THOMSON; SIBCLAIR, 2018).

Dessa forma, o estudo feito pelo biólogo da UnB, Marcelo Hermes-Lima, esclarece que "A melhor forma de analisar a qualidade de um conjunto grande de artigos é por meio de suas citações," (HERMES-LIMA, 2017).

\subsection{Análise da Tecnologia Considerando Número de Pedidos de Patentes Concedidas e Publicações}

A análise da razão entre patentes e publicações é importante para determinar a maturidade da tecnologia. Para tanto, foi utilizada para a busca a base de patentes Orbit e a base de artigos Web of Science.

A comparação entre as duas bases de artigos Scopus e Web of Science, realizada no presente trabalho em um primeiro momento, serviu para validar a informação encontrada de que existem mais pedidos de patentes do que produções científicas sobre a tecnologia estudada. Isso se dá pelo fato de existirem empresas investindo em tecnologias com foco no mercado e menos estudos publicados por universidades e demais instituições, possibilitando assim uma crescente comercialização de produtos nessa área. Tal situação é peculiar e denota que a maturidade da tecnologia é bem propícia ao mercado, tendo em vista que a publicação de artigos torna o conhecimento de domínio público, enquanto a patente traz a apropriação daquela tecnologia restringindo os direitos de exploração comercial do ativo exclusivamente ao detentor da patente. Esse crescimento relacionado aos depósitos de patentes também é típico de uma tecnologia emergente em seus primeiros anos de apropriação (QUINTELLA et al., 2011), conforme a Figura 7.

Figura 7 - Relação patentes x publicações para as palavras-chave "bioelec*" or "bio-signal" and "signal" and "body"

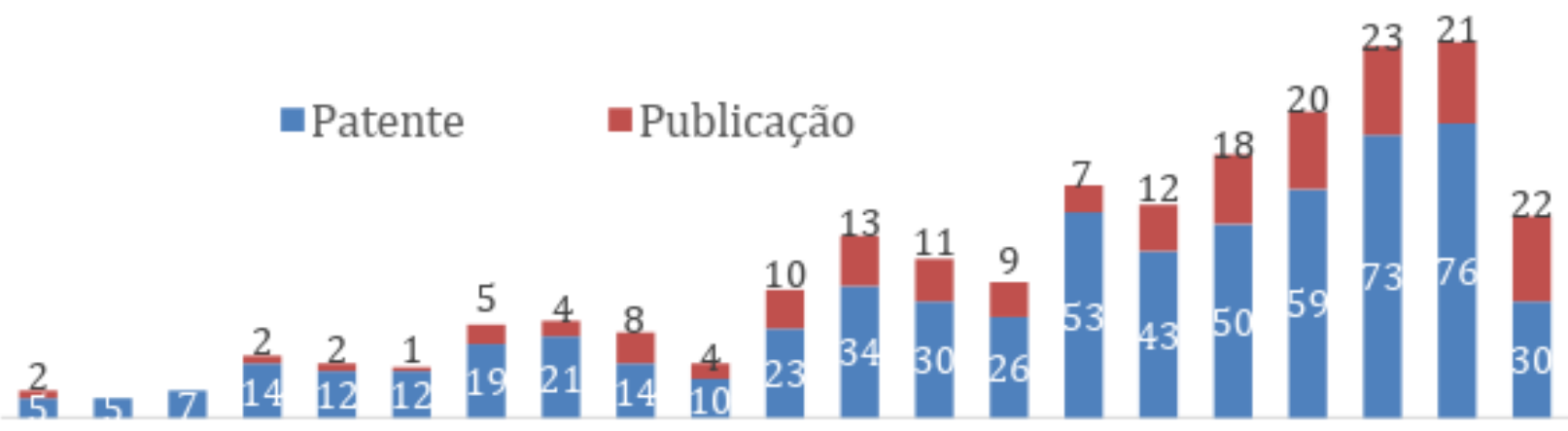

199719981999200020012002200320042005200620072008200920102011201220132014201520162017

Fonte: Elaborada pelos autores deste artigo (2018) 


\subsection{Análise da Tecnologia Utilizando o Método TRL}

O primeiro documento que descreveu a ideia sobre os níveis de maturidade das tecnologias foi o The NASA technology push towards future space mission systems (SADEN, 1989). Segundo Mankins (1995), por meio de um sistema de medição sistemática, os TRLs auxiliam as avaliações da maturidade de uma tecnologia em particular e também a comparação de maturidade entre diferentes tipos de tecnologia.

O método TRL é apresentado como uma ferramenta de política de pesquisa e inovação pelo The TRL Scale as a Reserch \& Innovation Policy Tool (EARTO, 2014), capaz de estimar a maturidade tecnológica dos elementos críticos da tecnologia durante o seu processo de aquisição e permitir discussões consistentes e uniformes de maturidade técnica em diferentes tipos de tecnologia.

A Resolução n. 191, de 18 de maio de 2017, do INPI, que institui o Projeto Piloto de Priorização do Exame de Pedidos de Patente depositados por Instituições de Ciência e Tecnologia, em seu artigo $2^{\circ}$, define TRL ou níveis de prontidão tecnológica como "[...] metodologia de estimação da maturidade tecnológica dos Elementos Tecnológicos Críticos (CTE) de um projeto através do processo de desenvolvimento, baseado em uma escala de 1 a 9". (BRASIL, 2017).

Com base nas concepções antes mencionadas e no modelo da referida resolução, que fornece uma sugestão de descrição e dos resultados dos TRLs, bem como na norma ISO 16290:2013, foi realizada a análise pelos autores de forma a terminar o grau de maturidade da tecnologia intitulada "palmilha sensorizada para pés diabéticos".

Inicialmente, verificou-se que a tecnologia sob análise demonstra, de forma simples, o processo tecnológico bem como expressa os princípios básicos destinados ao uso e aplicações potenciais. Os conceitos e a aplicação do produto são bem formulados, destinando-se especialmente para pessoas diabéticas, com confecção do produto de forma individualizada e personalizada.

O estabelecimento de função crítica, de forma analítica ou experimental, ou prova de conceito é demonstrada em publicações de pesquisas realizadas pelos professores responsáveis pela tecnologia e seus alunos. De acordo com o artigo Embodiments, Visualizations and Immersion with Enactive Affective Systems (MIT, 2017), a viabilidade do produto é ressaltada pela utilização de sensores apenas nos pontos específicos desejados.

A validação funcional dos componentes em ambiente de laboratório foi realizada apenas em parte. O estudo "Mathematical Modeling of Passive Diabetic Step" (FLEURY ROSA et al., 2014) mostra que a modelagem da marcha diabética é desafiadora e requer um estudo da marcha do paciente com diabetes para confecção da palmilha. Diante disso, o referido artigo retrata ainda que estão sendo realizados estudos e testes laboratoriais sobre a marcha de pessoas acometidas da doença por meio de um sistema que utiliza uma palmilha em látex com dispositivos sensoriais que identificam a pressão plantar nessas pessoas.

Da leitura do artigo Walking and health: an anctive affective system (LUCENA et al., 2016) depreende-se que uma das inventoras, a Prof. Suélia Rodrigues (BioEngLab/UnB), criou um biomaterial de palmilha que foi testado, para sinais vitais, como um tipo de assistente pessoal. Esse protótipo tem sido usado com sucesso para medir a pressão do pé, no intuito de servir como assistente de saúde, especialmente para pessoas diabéticas. $\mathrm{O}$ artigo supramencionado ainda relata que o protótipo pode ser considerado como uma inovação disruptiva aplicada para a saúde móvel. 
A validação e demonstração das funções críticas dos componentes em ambiente relevante foram realizadas. O protótipo foi reproduzido com comprovação de sua funcionalidade e desempenho. Contudo, não houve demonstração em ambiente operacional. Assim, necessário enfatizar que o produto não foi finalizado e qualificado, bem como não se encontra apto a ser comercializado.

Diante dessa análise, foi possível concluir que a tecnologia "palmilha sensorizada para pés diabéticos" ainda se encontra em fase de teste com nível de prontidão passível de ser classificado como TRL 5, que se refere à validação das funções críticas dos componentes em ambiente relevante. A prontidão da tecnologia ainda é muito incipiente, carecendo da realização de testes de efetividade em pessoas diabéticas.

É importante destacar que o grau de prontidão foi analisado com base nas informações constantes no pedido de patente depositado e em dados resultantes de estudos posteriores que estão disponíveis em publicações de autoria dos próprios inventores, tendo sido, portanto, consideradas as evoluções divulgadas publicamente nos últimos sete anos.

Vale ressaltar que as tecnologias com potencial de aplicação ao Sistema Único de Saúde (SUS) devem passar por um processo de certificação pela Anvisa em atendimento ao disposto na Lei n. 6.360/1976, artigo 12, que dispõe que nenhum produto de interesse à saúde, seja nacional ou importado, poderá ser industrializado ou comercializado no mercado brasileiro sem obter o registro junto ao Ministério da Saúde. Diante disso, faz-se necessário mencionar que a tecnologia em questão, carece de um longo caminho a ser percorrido até chegar ao mercado brasileiro, necessitando de realização de testes em pessoas acometidas pela diabetes, autorização de comercialização pelo Ministério da Saúde, produção de lote piloto e prototipagem a nível industrial. Ou seja, apesar de se tratar de uma tecnologia em elevado estado de prontidão, se comparado com grande parte das tecnologias desenvolvidas em universidades, esta ainda demandará um volume significativo de recursos de um eventual interessado antes que possa ser comercializado.

\section{Considerações Finais}

O estudo prospectivo conduzido pelo presente trabalho visou a identificar o mercado mundial em que a tecnologia "palmilha sensorizada para pés diabéticos" encontra-se inserida.

No presente trabalho foi apresentada uma prospecção, em nível nacional e internacional, da tecnologia de titularidade da FUB, detectando inventos similares, publicações sobre o assunto, viabilidade comercial bem como grau de prontidão tecnológica.

Utilizando-se as bases de dados das plataformas Orbit, Scopus e Web of Science, pode-se concluir a predominância de documentos de pedidos de patente, que perfazem o triplo do número de publicações científicas.

As informações levantadas tanto de patentes como de publicações indicam que os desenvolvimentos tecnológicos na área se encontram em crescimento, com ápice em 2014 e 2015, e que a maior parte das tecnologias similares estão sendo produzidas na Coréia do Sul. Também são realizados muitos estudos na Índia, EUA, Japão, Suíça, China e Itália. A empresa que mais desenvolve tecnologia na área é a Samsung. 
O fato de haver mais documentos publicados em papéis de conferência do que em artigos indica que a área precisa ainda ser mais bem consolidada cientificamente.

A classificação da tecnologia em TRL 5 evidenciou a necessidade do estabelecimento de parcerias estratégicas para o aprimoramento do produto, realização de testes em pessoas diabéticas e certificação na ANVISA.

As informações reunidas também mostraram ser possível a realização de parcerias para a futura comercialização do produto, haja vista o crescente número de brasileiros diagnosticados com a doença e a existência de indústrias atuantes na área de tecnologias para monitoramento, no corpo ou em suas partes, de sinais bioelétricos.

Conclui-se que a manutenção do pedido de proteção da patente e suporte dos gastos diretos do CDT/UnB efetuados ao INPI a título de taxas, se justificam.

O atual cenário no qual se localiza a tecnologia prospectada, ainda, é crescente e capaz de absorver e receber a invenção analisada.

\section{Referências}

ANDREASSI, T.; SBRAGI, R. Relações entre indicadores de P\&D e de resultado empresarial. Revista de Administração, São Paulo, v. 37, n. 1, p. 72-84, jan./mar. 2002.

ASSOCIAÇÃO BRASILEIRA DE NORMAS TÉCNICAS (ABNT). NBR ISO 16290: sistemas espaciais - definição dos níveis de maturidade da tecnologia (TRL) e de seus critérios de avaliação. Rio de Janeiro, 2013.

BIOELECTROMAGNETICS. Revista Científica, [S.l.], v. 39, n. 4, maio 2018. Disponível em: $<$ https://www.bems.org/node/15399>. Acesso em: 12 jun. 2018.

BOUYSSOU, D.; MARCHANT, T. Ranking scientists and departments in a consistent manner. Journal of the American Society for Information Science and Technology, [S.I.], v. 62, n. 9, p, $1.761-1.769,2011$.

BRASIL. Emenda Constitucional n. 95, de 16 de dezembro de 2016. Altera o Ato das Disposições Constitucionais Transitórias, para instituir o Novo Regime Fiscal, e dá outras providências. [2016]. Disponível em: < http://www.planalto.gov.br/ccivil_03/constituicao/emendas/ emc/emc95.htm >. Acesso em: 19 jun. 2018.

Lei n. 6.360, de 23 de setembro de 1976. Dispõe sobre a Vigilância Sanitária a que ficam sujeitos os Medicamentos, as Drogas, os Insumos Farmacêuticos e Correlatos, Cosméticos, Saneantes e Outros Produtos, e dá outras Providências. [1976]. Disponível em: <http://www. planalto.gov.br/ccivil_03/leis/L6360.htm>. Acesso em: 13 jun. 2018.

Resolução/INPI n. 191, de 18 de maio de 2017. Institui o Projeto Piloto de priorização do exame de pedidos de patentes depositados por Instituições de Ciência e Tecnologia, Patentes ICTs. [2017]. Disponível em: < http://www.inpi.gov.br/sobre/legislacao-1/ Resoluo1912017PatentesICTs.pdf>. Acesso em: 15 jun. 2018.

BRASIL. Secretaria de Vigilância em Saúde. Departamento de Vigilância de Doenças e Agravos não Transmissíveis e Promoção da Saúde. Vigitel Brasil 2016. Brasília, DF: Ministério da Saúde, 2017. Disponível em: <http://portalarquivos2.saude.gov.br/images/pdf/2018/marco/02/vigitel-brasil-2016. pdf $>$. Acesso em: 13 jun. 2018. 
CROSS, Di; THOMSON, S.; SIBCLAIR, A. Research in Brazil: a report for Capes by Clarivate Analytics, 2018. Disponível em: <http:/www.capes.gov.br/images/stories/download/ diversos/17012018-CAPES-InCitesReport-Final.pdf>. Acesso em: 15 jun. 2018.

QUINTELLA, C. M. et al. Captura de CO2: mapeamento tecnológico da captura de CO2 baseada em patentes e artigos. Salvador: EDUFBA, 2011.

DAIM, T. U. et al. Forecasting emerging Technologies: Use of bibliometrics and patente analysis. Technological Forecasting and Social Change, [S.I.], v. 73, p. 981-1.012, 2006.

EARTO Recommendations. The TRL scale as a reserch \& innovation policy tool. 2014. Disponível em: <http://www.earto.eu/fileadmin/content/03_Publications/The_TRL_Scale_as_a_R_I_ Policy_Tool_-_EARTO_Recommendations___Final.pdf>. Acesso em: 15 jun. 2018.

FLEURY ROSA, S. et al. 2014. Mathematical Modeling of Passive Diabetic Step. In: CONFERENCE: CONGRESSO NACIONAL DE ENGENHARIA MECÂNICA (CONEM), VIII., 2014. Uberlândia. 2014. Disponível em: <https://www.researchgate.net/publication/264672451_MATHEMATICAL_ MODELING_OF_PASSIVE_DIABETIC_STEP>. Acesso em: 13 jun. 2018.

HERMES-LIMA, M. Aumento da produção científica. 2017. Disponível em: < https://www1. folha.uol.com.br/ciencia/2017/10/1927163-brasil-aumenta-producao-cientifica-mas-impacto-dostrabalhos-diminui.shtml>. Acesso em: 12 jun. 2018.

INSTITUTO DE ENGENHEIROS ELETRICISTAS E ELETRÔNICOS (IEEE). [2018]. Disponível em: $<$ https://www.embs.org/>. Acesso em: 15 jun. 2018.

LUCENA, T. et al. Walking and health: an enactive affective system, Digital Creativity, [S.l.], v. 27, n. 4, p. 314-333, 2016. Disponível em: <https://doi.org/10.1080/14626268.2016.1262430>. Acesso em: 13 jun. 2018.

MANKINS, J. C. Technology readiness levels - A white paper, advanced concepts office - Office of space acess and technology. 1995. Disponível em: <http:/www.artemisinnovation.com/images/ TRL_White_Paper_2004-Edited.pdf>. Acesso em: 15 jun. 2018.

MERIGÓ, J. M.; GIL-LAFUENTE, A. M.; YQGER, R. R. An overview of fuzzy research with bibliometric indicators. Applied Soft Computing, [S.l.], v. 27, p. 420-433, 2015.

NATURE - Revista Científica. Estudo da National Science Foundation (NSF) dos Estados Unidos. 2018. Disponível em: <https:/www.ufrgs.br/blogdabc/china-e-o-pais-que-produz-maisartigos-cientificos-no-mundo-brasil-e-o-12o/ > . Acesso em: 12 jun. 2018.

ORBIT INTELLIGENCE. Questel Orbit: base de dados on-line. [2018]. Disponível em: <https:// www.orbit.com>. Acesso em: 14 jun. 2018.

REIS, M. do C. dos et al. FUB Palmilha sensorizada para pés diabéticos. RPI 2219, 2013. PI 1103691-5 A2, 18 jul. 2011, 16 jul. 2013.

PRITCHARD, A. Statistical bibliography or bibliometricas? Journal of Documentation, [S.l.], v. 25, n. 4, p. 348-349, dez. 1969.

SCOPUS. Base de dados on-line. [2018]. Disponível em: <https://www.scopus.com/home.uri>. Acesso em: 13 jun. 2018.

SERAFINI, M. R. et al. Mapeamento de tecnologias patenteáveis com o uso da hecogenina. Revista Geintec, [S.I.], v. 2, n. 5, p. 427-435, 2012. 
WEB OF SCIENCE. Base de dados on-line. [2018]. Coleção Principal. Thomson Reuters Scientific. Disponível em: <http://www.webofknowledge.com>. Acesso em: 15 jun. 2018.

\section{Sobre os Autores}

\section{Luciana Maria de Oliveira Cortinhas}

E-mail: luciana2610@yahoo.com.br

Formação: Mestranda no Programa de Pós-Graduação em Propriedade Intelectual e Transferência de Tecnologia para Inovação (PROFNIT), pela Universidade de Brasília (UnB); especialista em Direito Público, pela Universidade Sul de Santa Catarina (UNISUL); graduada em Direito, pela Pontifícia Universidade Católica do Paraná (PUC-PR); graduada em Artes Gráficas, pela Universidade Tecnológica Federal do Paraná (UTFPR).

Endereço profissional: Auditoria Interna da Universidade de Brasília. Campus Universitário Darcy Ribeiro, BAES. $1^{\circ}$ andar, Asa Norte - Brasília, DF.

\section{Leonara Gonçalves e Silva Pires}

E-mail: leonara.gs@gmail.com

Formação: Mestranda no Programa de Pós-Graduação em Propriedade Intelectual e Transferência de Tecnologia para Inovação (PROFNIT), pela Universidade de Brasília (UnB); especialista em Direito Público, pela UNIASSELVI; graduada em Direito, pela UDF.

Endereço profissional: Universidade de Brasília. Centro de Apoio ao Desenvolvimento Tecnológico. Campus Universitário Darcy Ribeiro, Edifício CDT, Asa Norte - Brasília, DF.

\section{Anna Patrícia Teixeira Barbosa}

E-mail: annapbarbosa@gmail.com

Formação: Mestranda no Programa de Pós-Graduação em Propriedade Intelectual e Transferência de Tecnologia para Inovação (PROFNIT), pela Universidade de Brasília (UnB); MBA em Gestão de Projetos, pela Universidade Católica de Brasília (UCB); graduada em Administração de Empresas, pelo Centro Universitário de Brasília (UNICEUB).

Endereço profissional: Sebrae Nacional - SGAS 605, lote A - Brasília, DF.

\section{Jeane Souza Chaves Sidou}

E-mail: jeanesidou@gmail.com

Formação: Mestranda no Programa de Pós-Graduação em Propriedade Intelectual e Transferência de Tecnologia para Inovação (PROFNIT), pela Universidade de Brasília (UnB); MBAs Propaganda e Marketing, Marketing de Serviço, Marketing organizacional e Administração Estratégica; graduada em História e Marketing.

Endereço profissional: Sidou Marketing \& Consultoria: SHN, Q. 05 Bl I, Sala 11 - Hotel Mercure Líder - Brasília, DF.

\section{Camila Alves Areda}

E-mail: caareda@unb.br

Formação: Doutora em Ciências Farmacêuticas, pela Universidade de São Paulo (USP); graduada em Farmácia Bioquímica, pela USP.

Endereço profissional: Universidade de Brasília. Campus Ceilândia - Ceilândia, DF. 


\section{Paulo Gustavo Barboni Dantas Nascimento}

E-mail: pbarboni@unb.br

Formação: Pós-doutor em Farmacologia, pela Universidade de São Paulo (USP); doutor, mestre e graduado em Química, pela Universidade de São Paulo (USP).

Endereço profissional: Universidade de Brasília. Campus Ceilândia - Ceilândia, DF.

\section{Rafael Leite Pinto de Andrade}

E-mail: rafael.andrade@cnpq.br

Formação: Doutor em Desenvolvimento, Agricultura e Sociedade, pela Universidade Rural do Rio de Janeiro (UFRRJ); mestrado em Ciência e Tecnologia de Alimentos, pela UFRRJ; especialista em Produção Audiovisual, pela Faculdade de Ciências Sociais e Tecnológicas (FACITEC); graduado em Engenharia de Alimentos, pela UFRRJ.

Endereço profissional: Conselho Nacional de Desenvolvimento Científico e Tecnológico. Brasília, DF. 